\title{
Analysis of double stub tuner control stability in a phased array antenna with strong cross-coupling
}

\author{
G.M. Wallace, P. Koert, Y. Lin, S. Shiraiwa, S.J. Wukitch \\ MIT Plasma Science and Fusion Center, Cambridge, MA 02139 USA
}

J. Hillairet

CEA-IRFM, Saint-Paul-lez-Durance, France

\begin{abstract}
Active stub tuning with a fast ferrite tuner (FFT) has greatly increased the effectiveness of fusion ion cyclotron range of frequency (ICRF) systems (50$100 \mathrm{MHz}$ ) by allowing for the antenna system to respond dynamically to changes in the plasma load impedance such as during the L-H transition or edge localized modes (ELMs). A high power waveguide double-stub tuner is under development for use with the Alcator C-Mod lower hybrid current drive (LHCD) system at $4.6 \mathrm{GHz}$. The amplitude and relative phase shift between adjacent columns of an LHCD antenna are critical for control of the launched $n_{\|}$spectrum. Adding a double-stub tuning network will perturb the phase and amplitude of the forward wave particularly if the unmatched reflection coefficient is high. This effect can be compensated by adjusting the phase of the low power microwave drive for each klystron amplifier. Crosscoupling of the reflected power between columns of the launcher must also be considered. The problem is simulated by cascading a scattering matrix for the plasma provided by a linear coupling model with the measured launcher scattering matrix and that of the FFTs. The solution is advanced in an iterative manner similar to the time-dependent behavior of the real system. System performance is presented under a range of edge density conditions from under-dense to over-dense and a range of launched $n_{\|}$. Simulations predict power reflection coefficients $\left(\Gamma^{2}\right)$ of less than $1 \%$ with no contamination of the $n_{\|}$spectrum. Instability of the FFT tuning network can be problematic for certain plasma conditions and relative phasings, but reducing the control gain of the FFT network stabilizes the system.
\end{abstract}

Preprint submitted to Fusion Engineering and Design

July 23, 2014

(C) 2014. This manuscript version is made available under the Elsevier user license

http://www.elsevier.com/open-access/userlicense/1.0/ 
Keywords: LHCD, Stub Tuner, Alcator C-Mod, Lower Hybrid Current Drive, Microwave

\section{Introduction}

Stub tuning networks have been used to reduce power reflected from the plasma on many fusion experiments in the ion cyclotron range of frequencies $(<\sim 100 \mathrm{MHz})[1,2]$ but have not yet been deployed at higher frequencies. A double stub matching network with electronically controlled tuning stubs is under development for the lower hybrid current drive (LHCD) system on Alcator C-Mod [3, 4]. The multijunction concept employed in many LHCD experiments $[5,6,7,8,9]$ reduces reflected power passively through destructive interference of the reflected waves, but at the cost of $n_{\|}$spectrum control. The peak $n_{\|}$of a multijunction antenna is adjustable over a small range $\left(n_{\|, \text {peak }}=n_{\|, 0} \pm \delta n_{\|}\right.$where $\delta n_{\|} \sim 0.1$ for ITER and $\delta n_{\|} \sim 0.3$ for Tore Supra), but is not capable of larger changes in $n_{\|}$or use in reverse current operation. Furthermore, side lobes in the $n_{\|}$spectrum of a multijunction antenna tend to grow when the reflection coefficient at the waveguide mouth is large. An active matching network like a double stub tuner allows for complete control of the $n_{\|}$spectrum, in either the co- or counter-current direction, while reducing reflection coefficients to near zero.

The behavior of a double-stub tuner connected in series with a single mismatched load, $Z_{L}$, is a well known problem in microwave engineering [10]. The matching network will reduce the input reflection coefficient, $\Gamma_{i n}$, to zero for any load impedance outside the "forbidden region", and from $\Gamma_{i n}$ to $\Gamma_{i n}^{2}$ for load impedances inside the forbidden region. The problem is more complicated for a phased array LHCD antenna with strong cross-coupling between elements. The effective reflection coefficient for each element, $\Gamma_{n}$, is a function not only of the plasma density profile but also of the relative phase and amplitude of other nearby radiating elements. Mathematically, this can be expressed in terms of a series of matrix multiplication operations involving the scattering parameters of the plasma, antenna, and individual FFT elements. The mathematical system description is discussed in the next section of this paper, followed by simulation results of the FFT system behavior with realistic plasma profiles. 


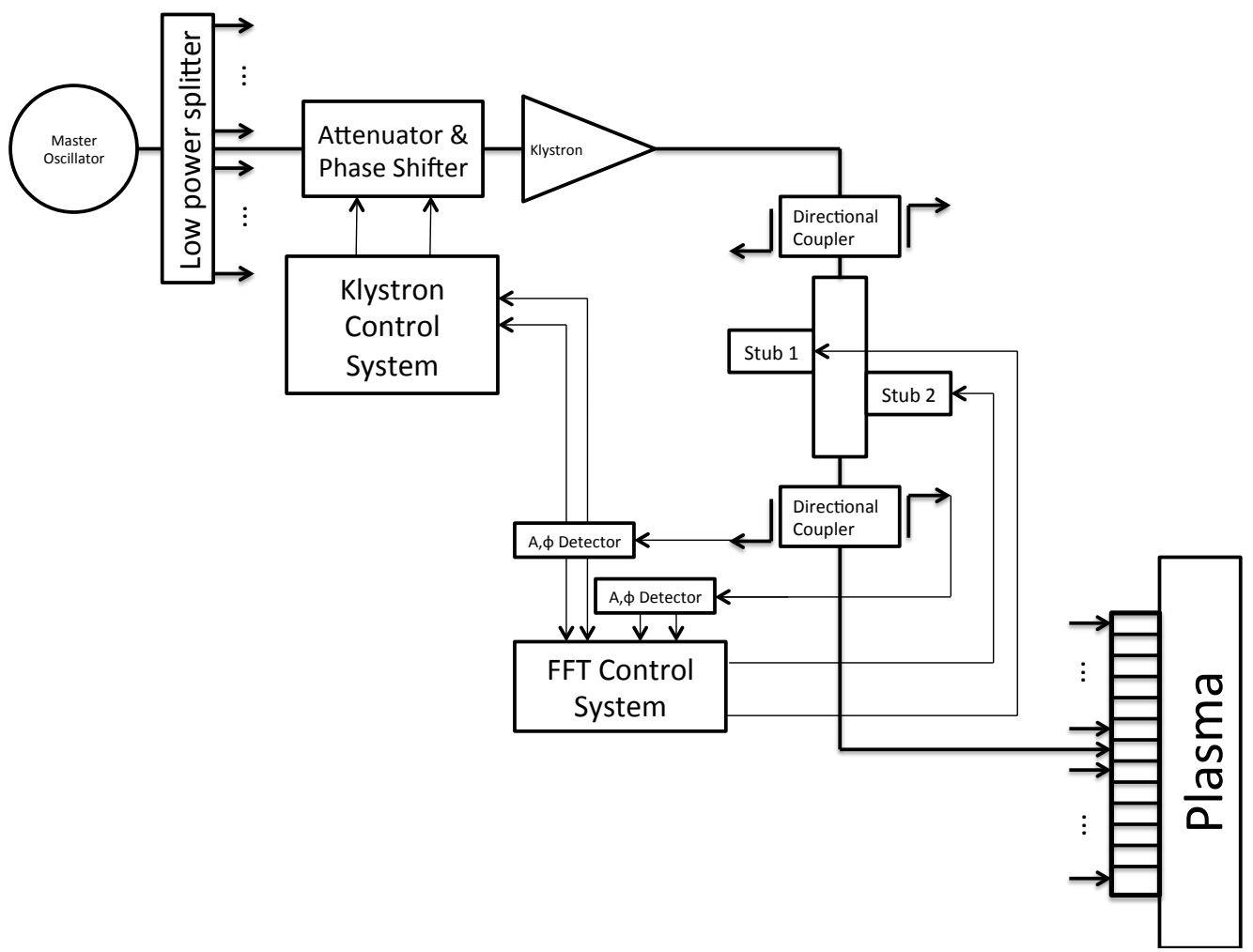

Figure 1: LH FFT system-level block diagram for a single klystron channel, or column. Each column is equipped with an identical system. Two tuning stubs are located between each klystron and the antenna.

\section{System description}

Figure 1 shows a block diagram for a single klystron channel of the LH FFT system. For the simulations presented in this paper, each klystron feeds a single column of a fully active "grill" antenna. The two stubs are located between the klystron and the antenna, with the stubs as close to the plasma as practical to minimize the region of recirculating power. Directional couplers for monitoring the forward and reflected power are located on either side of the LH FFT.

The plasma scattering matrix, $S_{p}$, can be calculated based on the linear coupling theory first developed by Brambilla [11]. The scattering matrix is determined by the geometry of the antenna (primarily the waveguide height and septum thickness) and the plasma profiles in front of the LH antenna 
(electron density, density gradient, and thickness of evanescent region). $S_{p}$ is an $n \times n$ matrix, where $n$ is the number of radiating elements in the antenna. The $(n, n)$ elements are of order 0.3 , while the $(n, n \pm 1)$ elements are of order 0.5 . The magnitude of matrix elements decreases farther away from the diagonal since radiating elements spaced farther apart have weaker cross-coupling. The magnitude and phase of $S_{p}$ for the fundamental mode as calculated by the ALOHA code [12] for a plasma with $n_{0}=2.7 \times 10^{17} \mathrm{~m}^{-3}$ and $\nabla n=4.7 \times 10^{20} \mathrm{~m}^{-4}$ are plotted in Figure 2. The pitch-angle of the magnetic field is not included in this calculation of $S_{p}$. Finite pitch angle will introduce non-reciprocity to the $S_{p}$ matrix.

The 2-port scattering matrix for a single FFT can be calculated given the reactive admittance of the two stubs, $B_{1}$ and $B_{2}$, the wave propagation constant of the waveguide, $\beta$, and the distance between the stubs, $l$. The most straightforward way to determine this scattering matrix is to convert from a series of three cascaded ABCD matrices

$$
A B C D_{F F T}=A B C D_{\text {stub } 2} A B C D_{\text {interstub }} A B C D_{\text {stub1 }}
$$

where

$$
\begin{aligned}
A B C D_{\text {stub } 2} & =\left[\begin{array}{cc}
1 & 0 \\
i B_{2} & 1
\end{array}\right] \\
A B C D_{\text {interstub }} & =\left[\begin{array}{cc}
\cos (\beta l) & i Z_{0} \sin (\beta l) \\
i Y_{0} \sin (\beta l) & \cos (\beta l)
\end{array}\right] \\
A B C D_{\text {stub } 1} & =\left[\begin{array}{cc}
1 & 0 \\
i B_{1} & 1
\end{array}\right]
\end{aligned}
$$

For simplicity we will assume $Z_{0}=Y_{0}=1$. From this ABCD matrix the S-matrix for the $k^{\text {th }}$ FFT can be calculated as follows

$$
S_{F F T k}=\left[\begin{array}{cc}
\frac{A+B / Z_{0}-C Z_{0}-D}{A+B / Z_{0}+C Z_{0}+D} & \frac{2(A D-B C)}{A+B / Z_{0}+C Z_{0}+D} \\
\frac{2}{A+B / Z_{0}+C Z_{0}+D} & \frac{-A+B / Z_{0}-C Z_{0}+D}{A+B / Z_{0}+C Z_{0}+D}
\end{array}\right]
$$

where $A, B, C, D$ are the four elements of the matrix $A B C D_{F F T}$.

The individual S-matrices for the $n$ FFTs are combined into a larger 

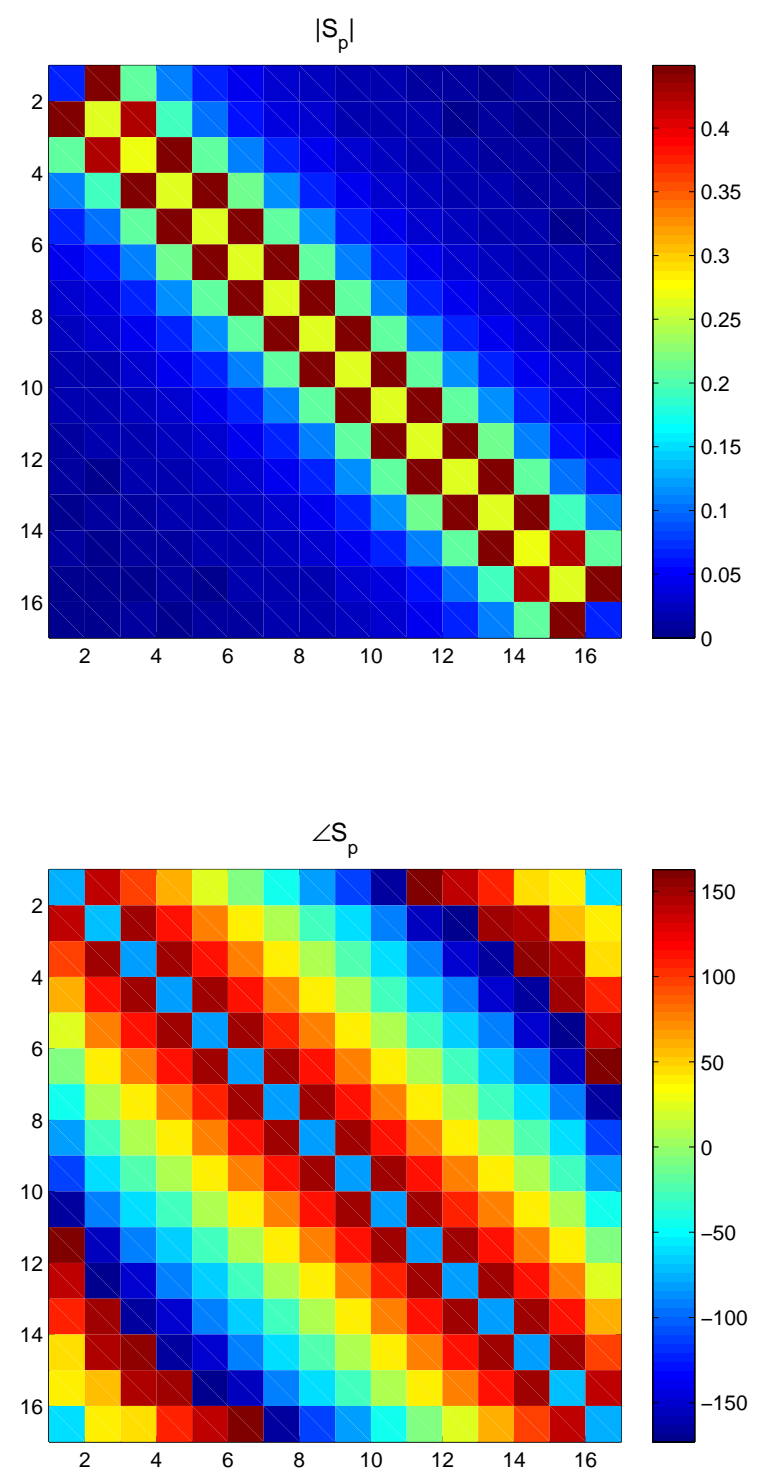

Figure 2: Magnitude and phase of the plasma scattering matrix, $S_{p}$, for $n_{0}=2.7 \times 10^{17} \mathrm{~m}^{-3}$ and $\nabla n=4.7 \times 10^{20} \mathrm{~m}^{-4}$. This S-matrix is calculated with the ALOHA coupling code $[12]$. 


\begin{tabular}{|c|c|c|c|c|c|c|c|c|}
\hline \multirow[b]{2}{*}[V_{in}(1)]{} & \multirow[b]{2}{*}{$\Gamma_{i n} \leftrightarrows$} & \multicolumn{7}{|c|}{$S_{\text {total }}$} \\
\hline & & {$\left[S_{F F T}(1,1)\right.$} & $\cdots$ & $S_{F F T}(1,2 n)$ & $\Gamma_{u} \leftrightarrows$ & $-\left[S_{p}(1,1)\right.$ & $\ldots$ & $\left.S_{p}(1, n)\right]$ \\
\hline & $\vdots$ & & $\because$ & & $\vdots$ & $\vdots$ & $\because$ & $\vdots$ \\
\hline$V_{i n}(n)$ & & {$\left[S_{F F T}(2 n, 1)\right.$} & $\cdots$ & $S_{F F T}(2 n, 2 n)$ & & $-S_{p}(n, 1)$ & $\cdots$ & $\left.S_{p}(n, n)\right]$ \\
\hline Input & & Stub $T t$ & ning & Network & & & Plasm & \\
\hline
\end{tabular}

Figure 3: A block diagram of the network connections between the plasma scattering matrix, $S_{p}$, the FFT scattering matrix, $S_{F F T}$, and the input power wave vector, $V_{i n}$.

$2 n \times 2 n$ S-matrix where

$$
\begin{aligned}
& S_{F F T}(k, k)=S_{F F T k}(2,2) \\
& S_{F F T}(k+n, k+n)=S_{F F T k}(1,1) \\
& S_{F F T}(k+n, k)=S_{F F T k}(1,2) \\
& S_{F F T}(k, k+n)=S_{F F T k}(2,1)
\end{aligned}
$$

for the $k^{\text {th }}$ FFT.

At this point, the two scattering matrices, $S_{p}$ and $S_{F F T}$, can be cascaded [13] to get the total scattering matrix, $S_{\text {total }}$. Figure 3 shows a block diagram of the cascaded system. Multiplying $S_{\text {total }}$ by the driving waveform power wave $(\mathrm{V} / \sqrt{\Omega})$ vector $V_{i n} \equiv \sqrt{P_{i n}}$ (e.g. $\{1, i,-1,-i, \ldots\}$ for $90^{\circ}$ phasing) gives the reflected wave for each input port of the combined $\mathrm{FFT} /$ plasma system. Since it is convenient to solve for the FFT stub lengths based on the reflection coefficient on the unmatched side of the FFT network, rather than the matched side, the unmatched reflection coefficient, $\Gamma_{u}$, must be calculated:

$$
\Gamma_{u}=S_{p} V_{\text {out }} \oslash V_{\text {out }}
$$

where $\oslash$ represents the element-wise division of the column vector $S_{p} V_{\text {out }}$ by the column vector $V_{\text {out }}$ and

$$
V_{\text {out }}=\left[I_{n_{\text {col }}}-S_{F F T}\left(1: n_{c o l}, 1: n_{c o l}\right) S_{p}\right]^{-1} S_{F F T}\left(1: n_{c o l}, n_{c o l}+1: 2 n_{c o l}\right) V_{\text {in }}
$$

Once $\Gamma_{u}$ is calculated, the necessary reactive admittance of the two FFT stubs can be determined analytically [10]:

$$
\begin{aligned}
& B_{1 k}=-B_{L}+\frac{Y_{0} \pm \sqrt{\left(1+t^{2}\right) G_{L} Y_{0}-G_{L}^{2} t^{2}}}{t} \\
& B_{2 k}=\frac{ \pm Y_{0} \sqrt{Y_{0} G_{L}\left(1+t^{2}\right)-G_{L}^{2} t^{2}}+G_{L} Y_{0}}{G_{L} t}
\end{aligned}
$$


Here, $Y_{k}=G_{L}+i B_{L}$ is the normalized unmatched load admittance corresponding to the $k^{\text {th }}$ element of $\Gamma_{u}$, and $t=\tan (\beta l)$. The electrical length of the short-circuited stub is:

$$
\frac{l_{s}}{\lambda}=\frac{-1}{2 \pi} \tan ^{-1}\left(\frac{Y_{0}}{B}\right)
$$

These new values for $B_{1}$ and $B_{2}$ are then inserted into Equation 2, and the process must be repeated. At each iteration $\Gamma_{u}$ may change based on the new values of $B_{1}$ and $B_{2}$ for each column.

\section{Simulation results}

The simulations presented in this paper begin with the stub lengths set for a matched load impedance. The system can be perfectly matched on the first iteration if the off-diagonal terms of $S_{p}$ are zero and the load does not lie within the forbidden region. For an LHCD launcher the cross-coupling between waveguides is significant (higher, in fact, than the diagonal elements of $S_{p}$ in most cases). In many cases the system converges quickly to a solution with very low reflection coefficients on the matched side, particularly when the off-diagonal terms of $S_{p}$ are weak or $n_{c o l} \leq \sim 4$. For larger, more coupled antenna arrays the system can be unstable if each FFT matching solution is calculated independently.

The behavior of the FFT/plasma system is studied here with a variety of plasma edge densities $\left(n_{c o}, 2 n_{c o}, 5 n_{c o}\right.$ where $n_{c o}=2.7 \times 10^{17}\left[\mathrm{~m}^{-3}\right]$ is the cutoff density for $4.6 \mathrm{GHz} \mathrm{LH}$ waves $)$, density gradients $\left(4.7 \times 10^{20}, 1.2 \times 10^{21}, 2.4 \times\right.$ $\left.10^{21}\left[\mathrm{~m}^{-4}\right]\right)$ and launched $n_{\|}\left(1.63,1.95,2.60,3.9\right.$; or $75^{\circ}, 90^{\circ}, 120^{\circ}, 180^{\circ}$ phasing). The plasma S-matrices used here are generated by the linear coupling code ALOHA [12]. Figure 4 shows the average matched reflection coefficient as a function of iteration number for a set of representative plasma conditions and phasings. The lowest and highest phasings exhibit unstable behavior under some conditions, while the reflection coefficient for moderate phasings drop quickly to very low levels $\left(\Gamma^{2}<10^{-4}\right)$.

The launched $n_{\|}$spectrum is also perturbed by the matching network. This effect can be compensated by adjusting $V_{i n}$ such that the magnitude and phase of $V_{\text {out }}$ matches that of the desired amplitude and phase for each

waveguide, $V_{\text {demand }}$. The magnitude and phase of the forward wave at each waveguide aperture are plotted in Figure 5. In this scenario the phase error due to the matching network is largely a linear offset to the demanded phase, 

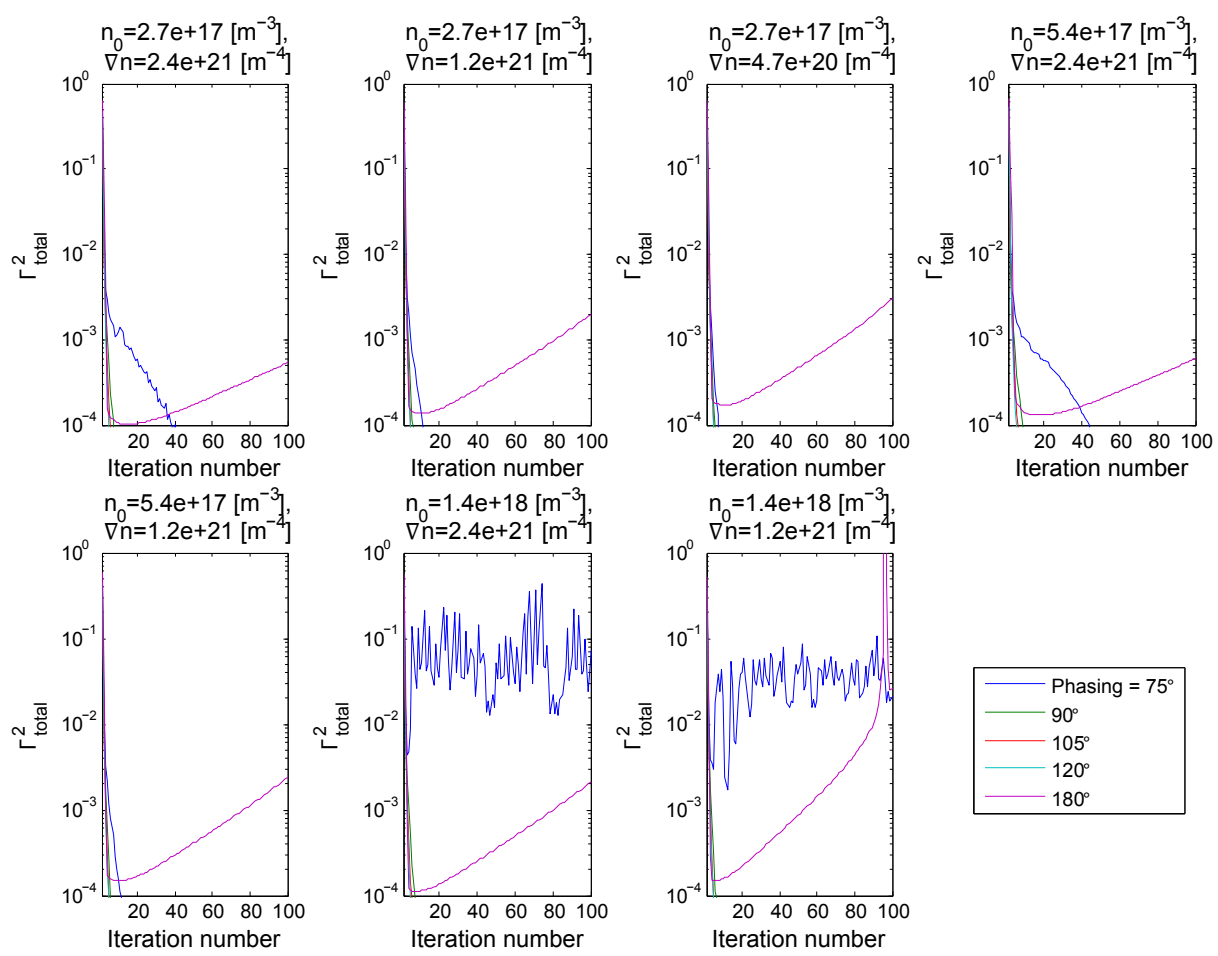

Figure 4: Overall power reflection coefficient $\Gamma^{2}$ of the LH FFT system for a single grill comprised of 16 columns with unity control system gain. The system is allowed to step forward for 100 time iterations. At each iteration a new set of FFT stub lengths is calculated and implemented based on the value of $\Gamma$ for each column in the previous iteration. The gain, $G$, is 1 for this simulation. 
but there is a noticeable increase in the amplitude of the forward wave for most columns. This adjustment can also introduce instability in some circumstances. Figure 6 shows the same information as in Figure 4 but with the addition of phase and amplitude compensation for the forward wave of each column. the compensation process introduces additional instability for $75^{\circ}$ and $90^{\circ}$ phasings across a range of plasma loading scenarios.

Reducing the "gain" of the FFT control system can help to stabilize the response. Here, the gain, $G$, is defined as:

$$
G=\frac{B_{n+1}-B_{n}}{B_{c a l c}-B_{n}}
$$

where $B_{n}$ is the stub reactive admittance at the $n^{\text {th }}$ iteration, $B_{n+1}$ is the programmed reactive admittance for the subsequent iteration, and $B_{\text {calc }}$ is the calculated "ideal" reactive admittance for the subsequent iteration. The value of $G$ represents the fractional amount of correction applied to the stub lengths between successive iterations. Figures 7 and 8 show the effect of reducing the gain without and with spectrum compensation, respectively. The iterative behavior is stable for all phasings and all plasma scenarios without spectrum compensation, and with spectrum compensation only $180^{\circ}$ "heating" phasing shows instability.

\section{Discussion}

The simulations show that system stability is dependent on both the plasma profiles and waveguide-to-waveguide phasing used, although it is not obvious from the governing equations why certain sets of density and phasing produce stable behavior while others are unstable. The $180^{\circ}$ case may be unstable due to the symmetry of the $\angle S_{p}$ matrix plotted in Figure 2. For a given waveguide, $N$, the cross coupling contributions from waveguides $N-1$ and $N+1$ will interfere constructively. It is important to note that the values used for $S_{p}$ do not include effects of magnetic field pitch-angle, and the results may change somewhat when these symmetry-breaking effects are included.

Decreasing the control system gain, $G$, is effectively equivalent to reducing the slew rate of the control coil current, or alternatively increasing the update rate of the reflection coefficient measurement/stub length calculation, with other parameters held constant. Effort has been expended to increase the slew rate of the electromagnet coil currents and decrease the penetration time 

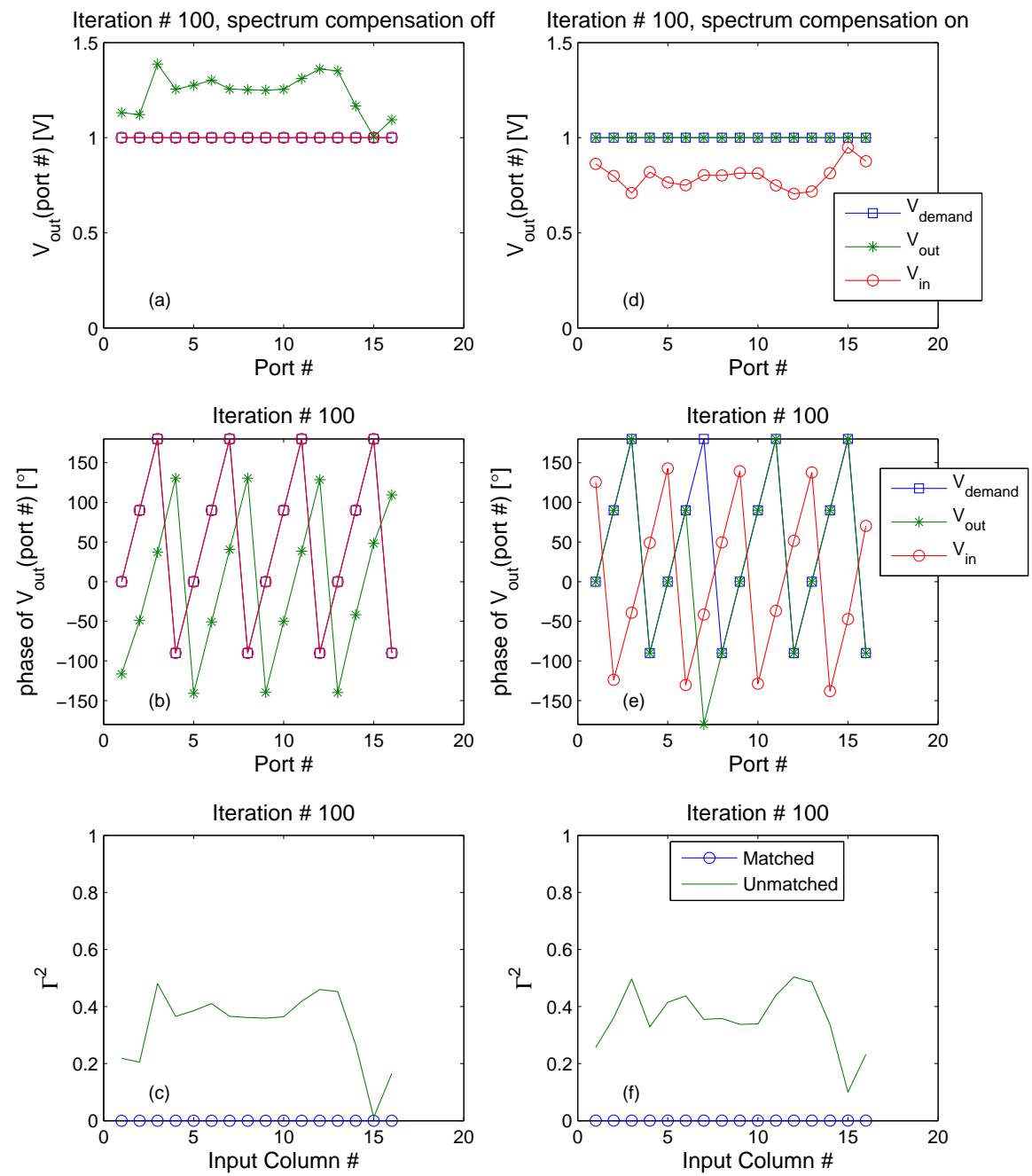

Figure 5: Magnitude and phase of the forward wave at the waveguide aperture without spectrum compensation and reflection coefficient for each column (a-c). Magnitude and phase of the forward wave at the waveguide aperture with spectrum compensation and reflection coefficient for each column (d-f). Simulations shown are for $90^{\circ}$ phasing with $n_{0}=2.7 \times 10^{17} \mathrm{~m}^{-3}$ and $\nabla n=4.7 \times 10^{20} \mathrm{~m}^{-4}$. 

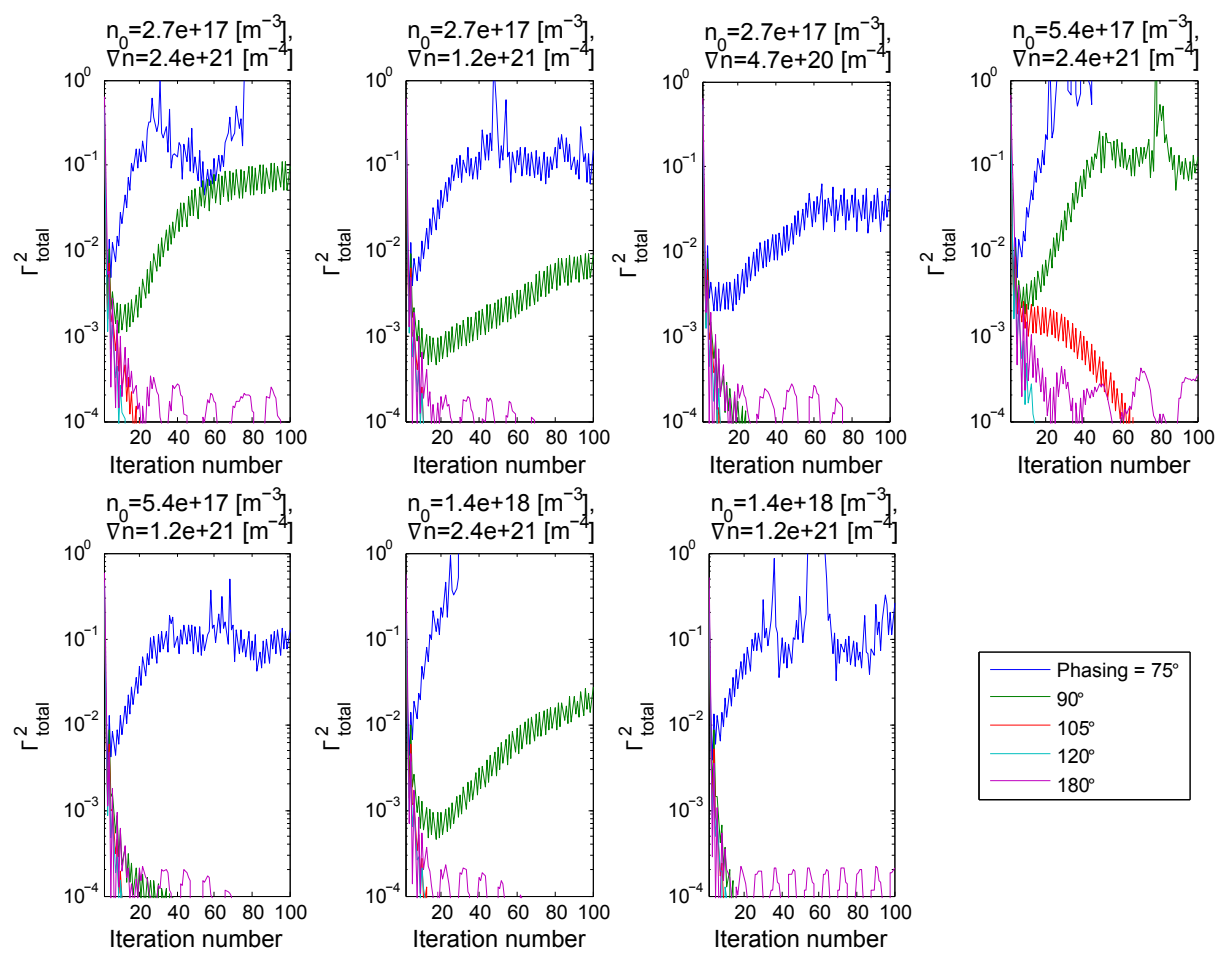

Figure 6: Overall power reflection coefficient $\Gamma^{2}$ of the LH FFT system for a single grill comprised of 16 columns with unity control system gain. The system is allowed to step forward for 100 time iterations. At each iteration a new set of FFT stub lengths is calculated and implemented based on the value of $\Gamma$ for each column in the previous iteration. The phase and amplitude of the forward wave for each column is compensated at every other iteration step. The gain, $G$, is 1 for this simulation. 

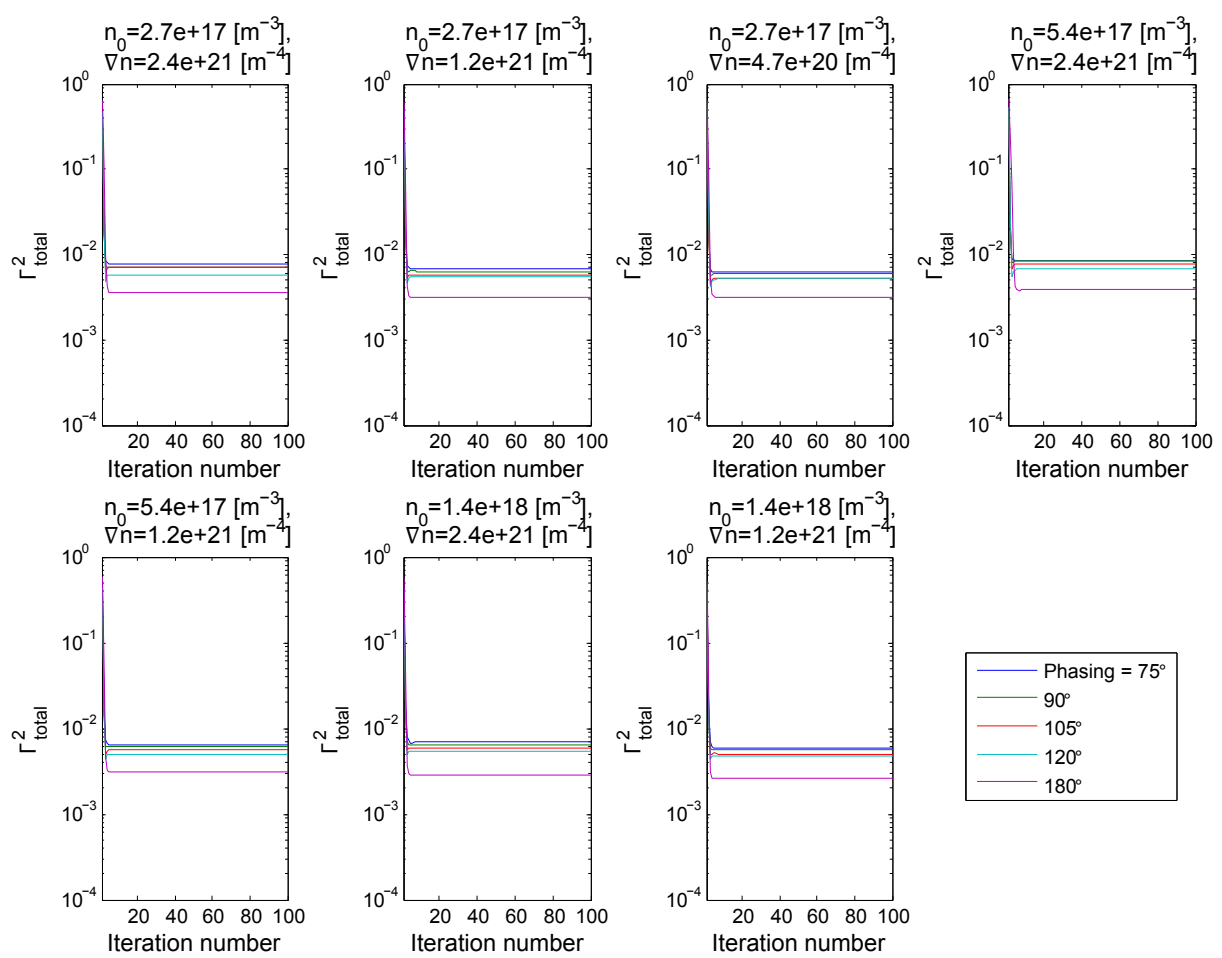

Figure 7: Overall power reflection coefficient $\Gamma^{2}$ of the LH FFT system for a single grill comprised of 16 columns. The system is allowed to step forward for 100 time iterations. At each iteration a new set of FFT stub lengths is calculated and implemented based on the value of $\Gamma$ for each column in the previous iteration. The gain, $G$, is 0.75 for this simulation. 


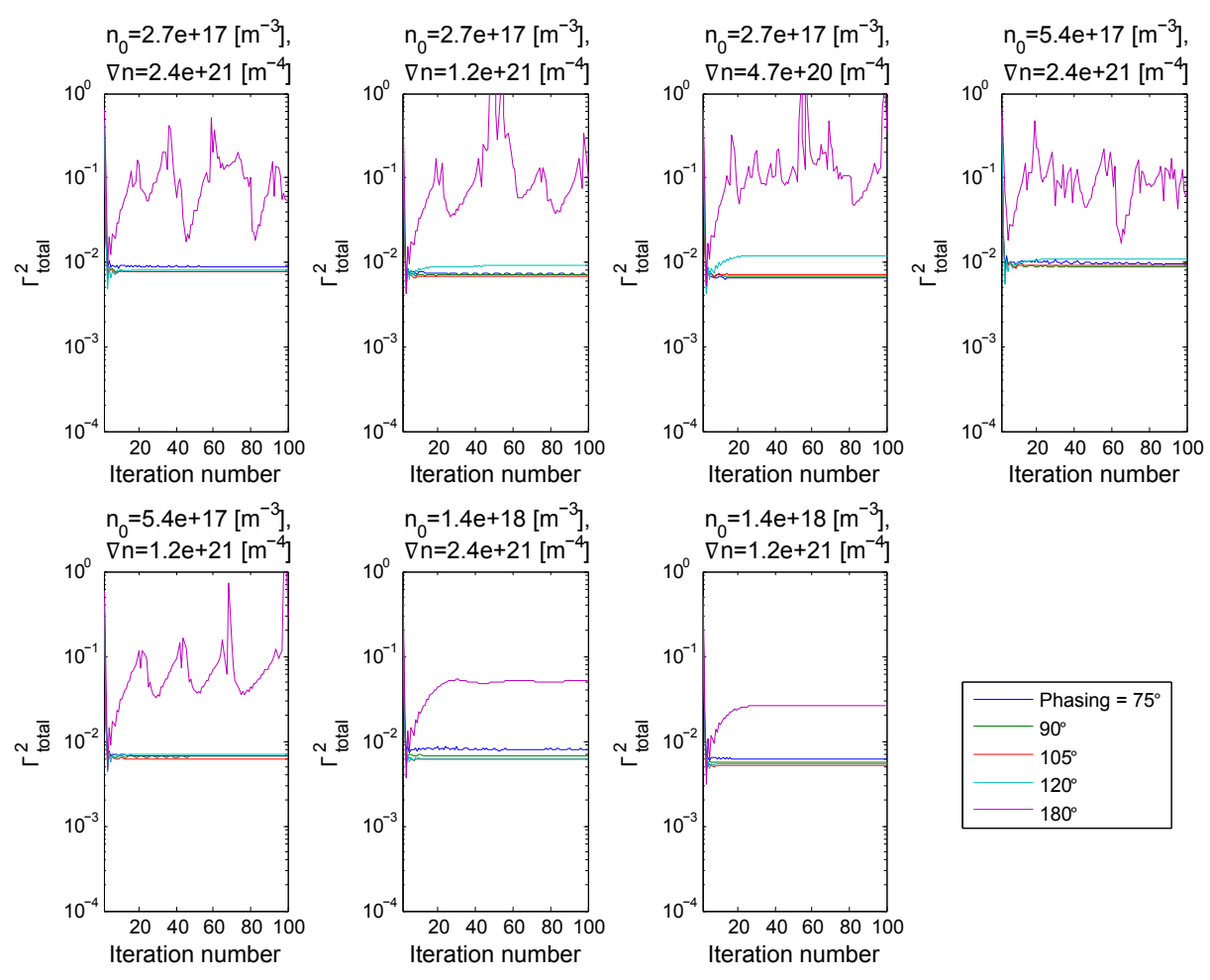

Figure 8: Overall power reflection coefficient $\Gamma^{2}$ of the LH FFT system for a single grill comprised of 16 columns. The system is allowed to step forward for 100 time iterations. At each iteration a new set of FFT stub lengths is calculated and implemented based on the value of $\Gamma$ for each column in the previous iteration. The phase and amplitude of the forward wave for each column is compensated at every other iteration step. The gain, $G$, is 0.75 for this simulation. 
for the resulting magnetic fields into the ferrite [14]. The simulations in this paper show that it may be necessary to slow down the response of the coils to avoid instability in the matching network.

Decreasing the gain has some negative impact on the final matched value of the reflection coefficient with an asymptote just under $\Gamma^{2}=10^{-2}$. With a gain of 1 and no spectrum compensation $\Gamma^{2}<10^{-4}$ in most cases. In practice a value of $10^{-2}$ is sufficiently low that the reflected power is still negligible so long as a circulator is used to protect the klystron amplifier from any reflected power.

The simulations presented in this paper consider only a single row of waveguides for the antenna, although in most LHCD experiments the antenna consists of several poloidal rows of waveguides. Adding multiple waveguide rows is not expected to substantively change the simulation results since the cross coupling between rows is very weak. Figure 2 shows that the crosscoupling between waveguides is very small for distant waveguides on the same field line (recall that the pitch-angle is zero in these simulations). The small magnetic field pitch-angle at the edge of a tokamak $\left(\sim 10^{\circ}\right)$ creates a long connection length between waveguide rows. For the dimensions of the C-Mod LHCD launcher $(7 \times 60 \mathrm{~mm}$ waveguides with a $1.5 \mathrm{~mm}$ septum between columns and a $20 \mathrm{~mm}$ separation between rows) the connection length between the two rows is $\sim 115 \mathrm{~mm}$, or $\sim 13.5$ columns. Figure 2 shows that the cross-coupling between waveguides at this distance within the same row is weak, and should also be weak for coupling between rows at this distance.

Although the simulations presented here are particular to the case of a LHCD phased array antenna, the simulation framework can be used to analyze stub-tuning performance in any phased array with cross-coupling between radiating elements. Only a new plasma scattering matrix, $S_{p}$, is required to adapt this simulation framework for analysis of other types of multi-element antennas with active double stub tuning (e.g. the ICRF or high harmonic fast wave (HHFW) regimes for fusion plasmas, or phased array radar systems) antennas. Most present generation ICRF antennas have a small number of radiating elements $(n \sim 4)$, which lessens the detrimental effect of cross coupling and can be addressed with a small number of decoupling loops in the air-side transmission line network. If the number of ICRF straps increases greatly it may be necessary to investigate the stability of the tuning network. 


\section{Summary}

A simulation model has been developed to assess the stability of a double stub tuner in a phased array with strong cross coupling. This model has been applied to the particular case of a LHCD antenna launching waves into a tokamak plasma at a frequency of $4.6 \mathrm{GHz}$. Simulations using a representative set of plasma scattering matrices, as computed by the ALOHA code, show that the LH FFT system is stable and produces clean $n_{\|}$spectra with good matching across a variety of plasma conditions and relative phasings. Reduction of the control system gain may be necessary for some conditions, although the decrease in system performance with reduced gain is acceptable.

\section{Acknowledgments}

This work supported by US Department of Energy cooperative agreement DE-FC02-99ER54512 using the Alcator C-Mod tokamak, a DOE Office of Science user facility. 


\section{References}

[1] R.I. Pinsker. Development of impedance matching technologies for icrf antenna arrays. Plasma Physics and Controlled Fusion, 40(8A):A215, 1998.

[2] Y. Lin, A. Binus, and S.J. Wukitch. Real-time fast ferrite ICRF tuning system on the Alcator C-Mod tokamak. Fusion Engineering and Design, 84(1):33-37, 2009.

[3] G.M. Wallace, S. Shiraiwa, J. Hillairet, M. Preynas, W. Beck, J.A. Casey, J. Doody, I.C. Faust, E. Fitzgerald, D.K. Johnson, A.D. Kanojia, P. Koert, C. Lau, Y. Lin, R. Leccacorvi, P. MacGibbon, O. Meneghini, R. Murray, R.R. Parker, D.R. Terry, R. Vieira, J.R. Wilson, S. Wukitch, and L. Zhou. Advances in lower hybrid current drive technology on alcator c-mod. Nuclear Fusion, 53(7):073012, 2013.

[4] P. Koert, D. Terry, E. Fitzgerald, P. MacGibbon, G. Wallace, and M. Takayasu. Development of fast ferrite tuner for lower hybrid current drive. In Fusion Engineering (SOFE), 2011 IEEE/NPSS 24th Symposium on, pages $1-4$, june 2011 .

[5] C. Gormezano, P. Briand, G. Briffod, G.T. Hoang, T.K. N'Guyen, D. Moreau, and G. Ray. Lower-hybrid plasma heating via a new launcher the multijunction grill. Nuclear Fusion, 25(4):419, 1985.

[6] Y. Ikeda, T. Imai, K. Ushigusa, M. Seki, K. Konishi, O. Naito, M. Honda, K. Kiyono, S. Maebara, T. Nagashima, M. Sawahata, K. Suganuma, N. Suzuki, K. Uehara, K. Yokokura, and JT-60 Team. Efficient lower hybrid current drive using a multijunction launcher on JT-60. $\mathrm{Nu}$ clear Fusion, 29(10):1815, 1989.

[7] X. Litaudon and D. Moreau. Coupling of slow waves near the lower hybrid frequency in JET. Nuclear Fusion, 30(3):471, 1990.

[8] X. Litaudon, G. Berger-by, P. Bibet, J. P. Bizarro, J. J. Capitain, J. Carrasco, M. Goniche, G. T. Hoang, K. Kupfer, R. Magne, D. Moreau, Y. Peysson, J.-M. Rax, G. Rey, D. Rigaud, and G. Tonon. Lower hybrid wave coupling in TORE SUPRA through multijunction launchers. Nuclear Fusion, 32:1883-1898, November 1992. 
[9] V. Pericoli Ridolfini, P. Bibet, F. Mirizzi, M. L. Apicella, E. Barbato, P. Buratti, G. Calabrò, A. Cardinali, G. Granucci, L. Panaccione, S. Podda, C. Sozzi, and A. A. Tuccillo. LHCD and coupling experiments with an ITER-like PAM launcher on the FTU tokamak. Nuclear Fusion, 45:1085-1093, September 2005.

[10] D.M. Pozar. Microwave Engineering. John Wiley \& Sons, Inc., 1998.

[11] M. Brambilla. Slow-wave launching at the lower hybrid frequency using a phased waveguide array. Nuclear Fusion, 16:47-54, February 1976.

[12] J. Hillairet, D. Voyer, A. Ekedahl, M. Goniche, M. Kazda, O. Meneghini, D. Milanesio, and M. Preynas. ALOHA: an Advanced LOwer Hybrid Antenna coupling code. Nuclear Fusion, 50(12):125010, 2010.

[13] G.R. Simpson. A generalized n-port cascade connection. In Microwave Symposium Digest, 1981 IEEE MTT-S International, pages 507-509, 1981.

[14] P. Koert, D.R. Terry, E. Fitzgerald, A.D. Kanojia, G.M. Wallace, R. Murray, and S.J. Wukitch. Operation of a double stub tuner for Alcator C-Mod lower hybrid current drive system. In 2013 Symposium on Fusion Engineering (SOFE), 2013. 